\title{
Alternative Inverted Middle Fossa Approach in Bonebridge Surgery. Technique, Results and Complications
}

\author{
Mario E. Zernotti ${ }^{1,2,3(-)}$ Maria F. Di Gregorio ${ }^{10}$ \\ ${ }^{1}$ Department of ENT, Universidad Nacional de Cordoba, Sanatorio \\ Allende, Cordoba, Córdoba, Argentina \\ 2 Department of Otorhinolaringology, Universidad Nacional de \\ Cordoba, Cordoba, Córdoba, Argentina \\ ${ }^{3}$ Department of Otorhinolaringology, Universidad Católica de \\ Cordoba, Cordoba, Córdoba, Argentina
}

\author{
Máximo Zernotti ${ }^{1}$
}

Int Arch Otorhinolaryngol 2021;25(3):e374-e378.

\begin{abstract}
Keywords

- conductive hearing loss

- aural atresia

- bone conductive hearing implant

Introduction The transmastoid approach is the most recommended technique to Bonebridge surgery, while in patients with bad anatomy or in the canal wall down technique, retrosigmoid or Middle Fossa Approaches are the alternative surgical options.

Objective To describe a novel alternative approach called inverted middle fossa approach (IMFA) and its technique and audiological outcomes.

Methods Seven patients submitted to the IMFA were included. All patients presented conductive and mixed hearing loss with bone thresholds of the audiogram $>40 \mathrm{~dB}$. The audiological test was conducted pre- and postoperatively.

Results A total of 5 males and 2 females, aged 13,8 years old (range $6-25$ years old) were studied. The average follow-up was of 20 months ( 12 to 32 months). All patients presented aural atresia, except one with severe osseous-fibrous dysplasia of the temporal bone. Two patients showed bilateral compromise, three patients had associated Goldenhar and Treacher Collins syndrome. On the preoperative audiograms, air conduction (AC) thresholds showed a PTA4 $(0.5,1,2$ and $4 \mathrm{kHz})$ of $66.7 \mathrm{~dB}$ (standard deviation $[S D]= \pm 7.8$ ), while the bone conduction thresholds reached an average of $11.2 \mathrm{~dB}$ ( $\mathrm{SD}= \pm 6.9$ ). The postoperative thresholds did not change, and additional sensorineural damage was not observed before activation. Four weeks after surgery, all the patients were fitted with the external processor. The postoperative audiological aided exam showed AC PTA 4 thresholds of $18.9 \mathrm{~dB}(\mathrm{SD}= \pm 5.9)$.

Conclusion The IMFA allows the nearest position of the microphone to the external auditory canal. The technique is a suitable option to the 3 classical approaches with similar rate of audiological results. More investigation is needed to determine the benefit of the novel approach compared with the others.
\end{abstract}

Address for correspondence Mario Zernotti, MD, PhD, Department of ENT, Universidad Nacional de Cordoba, Sanatorio Allende, 757 Independencia St., Cordoba 5000, Argentina (e-mail: mario.zernotti@gmail.com). received

May 3, 2020

accepted

June 24,2020

published online

September 24, 2020

\footnotetext{
September 24, 2020
}

DOI https://doi.org/ 10.1055/s-0040-1715152. ISSN 1809-9777.

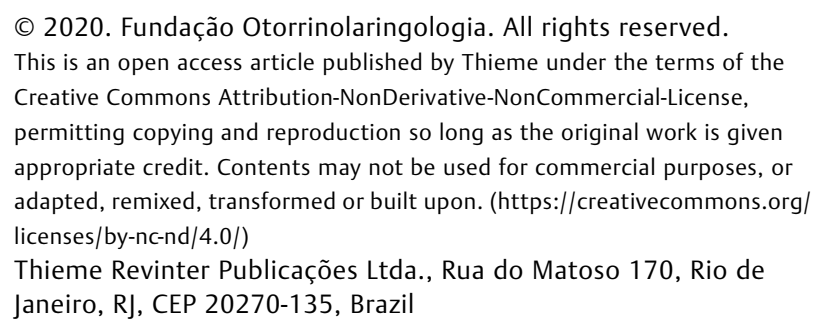
Creative Commons Attribution-NonDerivative-NonCommercial-License, permitting copying and reproduction so long as the original work is given appropriate credit. Contents may not be used for commercial purposes, or adapted, remixed, transformed or built upon. (https://creativecommons.org/ licenses/by-nc-nd/4.0/) Thieme Revinter Publicações Ltda., Rua do Matoso 170, Rio de Janeiro, RJ, CEP 20270-135, Brazil 


\section{Introduction}

Bone conduction hearing aids (BCHAs) can be classified in active or passive ones, and according to the status of the skin in transcutaneous or percutaneous. ${ }^{1}$ The first active transcutaneous BCHA (Bonebridge, Vibrant MED EL, Innsbruck, Austria) was approved for commercialization in 2012. Its main indication is the treatment of conductive or mixed hearing loss and single sided deafness (SSD). In our experience, and due to the high prevalence of congenital aural atresia (CAA) in our country, ${ }^{2}$ the most important indication is in patients suffering from this malformation, despite the fact that patients with chronic otitis media and otosclerosis are also usual candidates. The Bonebridge is a partially implanted bone conductive hearing system that consists in external and implanted components. The external part is the audio-processor (AP), including microphone and battery, while the implanted part is called bone conductive floating mass transducer (BC-FMT) and releases vibrations to the inner ear. Both components are coupled trough a magnet keeping the skin intact.

Originally, the manufacturer proposed the transmastoid approach except in patients without a good mastoid anatomy or in patients with radical cavities (canal wall down technique); in these patients, the proposed option is the retrosigmoid route (3-4-5). However, there are many patients for whom the placement of a Bonebridge. Therefore, in 2013, Agrawal described the technique of the middle fossa approach for these patients. $^{3-6}$

An alternative surgical approach is proposed in the present paper. This approach allows the placement of the device in very complex anatomies, such as patients presenting CAA. The originally described surgery uses the bone space above the temporalis line to insert the $\mathrm{BCI}$, with the external processor usually above the original position of the $\mathrm{BCI}$. This means that the microphone usually is far from the pinna.

The main objective of the present paper is to describe an alternative technique in the middle fossa approach, reversing the arrangement of the device to facilitate the placement of the external part of the device with a more appropriate position of the microphones, closer to the pinna and slightly over the horizontal axis of the external auditory canal (EAC). The natural microphone is the ear drum at the end of the EAC, therefore we proposed this new technique to imitate the nearest position to the natural microphone.

\section{Material and Methods}

This is a prospective, observational study approved by the institutional ethics committee and registered at the Health Ministry ( $\left.N^{\circ} 235 / 2018\right)$. Seven patients submitted to Bonebridge surgery using the IMFA technique were included. Most of them presented CAA, and one patient had osseofibrous dysplasia of the temporal bone.

The decision of the approach is based on the previous radiological planning. When neither the mastoid route nor the retro sigmoidal approach offered a better alternative than the middle fossa, the inverted middle fossa approach (IMFA) was performed.

\section{Inclusion Criteria}

Conductive and mixed hearing loss with a PTA4 audiogram showing $\mathrm{BC}$ thresholds $>40 \mathrm{~dB}$;

Patients $\geq 6$ years old;

Signed and dated informed consent.

All patients underwent the IMFA approach. All the surgeries were performed by the same surgeon. X-ray control was done immediately.

Minimum follow-up of 12 months.

\section{Audiological Evaluation Protocol}

Preoperative measurements of audiometry were performed, as well as free field audiometry in quiet and noise. Audiogram (hearing threshold) and word recognition scores were measured according to the following scheme. The first measurement was before surgery in unaided condition, and the day of switch on in unaided and aided condition, and 1 and 6 months later. Audiogram was done by warble tones in freefield using an Interacoustics AC- 40b clinical audiometer (Interacoustics, Middelfart, Denmark). The following frequencies were measured: 0.25, 0.5, 1, 2, 4 and $8 \mathrm{KHz}$;; while speech perception was evaluated using phonetically balanced disyllabic words in Argentinian Spanish (Tato list). The tests were performed in quiet and in noise (65 dB SPL using a loudspeaker and plugging the contralateral side when indicated).

\section{Results}

The IMFA was performed on a total of 7 patients. See - Table 1

The average age was 13.8 years old (range $6-25$ years old), 5 were male and 2 were female. All of the patients presented microtia and external and middle ear malformations, except only one with a severe osseofibrous dysplasia of the temporal bone. Two patients showed bilateral compromise of the atresia, three patients had associated syndromes, such as Goldenhar (two patients) and Treacher Collins. Follow-up occurred on average at 20 months, ranging from 12 to 32 months.

\section{Surgical Technique}

A semicircular incision is made around the $\mathrm{BCI}$, then a double layered flap of skin and muscle is done ( - Fig. 1 A-B). Inferiorly, a pocket is made to place the coil with the magnet, looking for

Table 1 Demographic and characteristics of patients

\begin{tabular}{|r|r|l|l|}
\hline $\mathbf{N}$ & \multicolumn{1}{|c|}{$\begin{array}{c}\text { Age at } \\
\text { surgery }\end{array}$} & Gender & Pathology \\
\hline 1 & 12 & female & bilateral microtia \\
\hline 2 & 13 & male & unilateral microtia \\
\hline 3 & 15 & male & osseous-fibrous dysplasia \\
\hline 4 & 11 & male & Goldenhar Syndrome \\
\hline 5 & 25 & male & unilateral microtia \\
\hline 6 & 6 & female & Goldenhar Syndrome \\
\hline 7 & 15 & male & Treacher Collins Syndrome \\
\hline
\end{tabular}



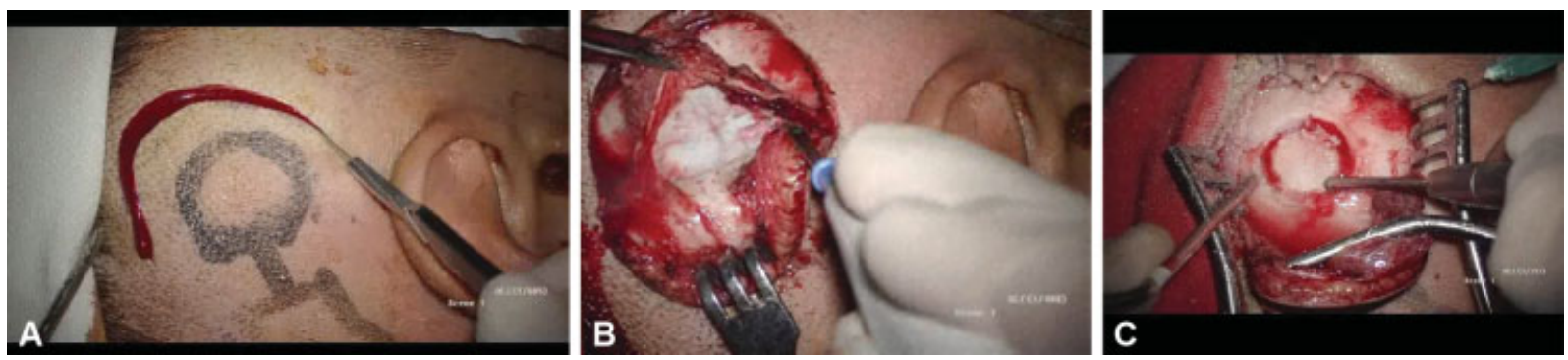

Fig. 1 (A) Semicircular incision above the pinna, (B): double layered flap, skin and muscular one, (C): implant bed.
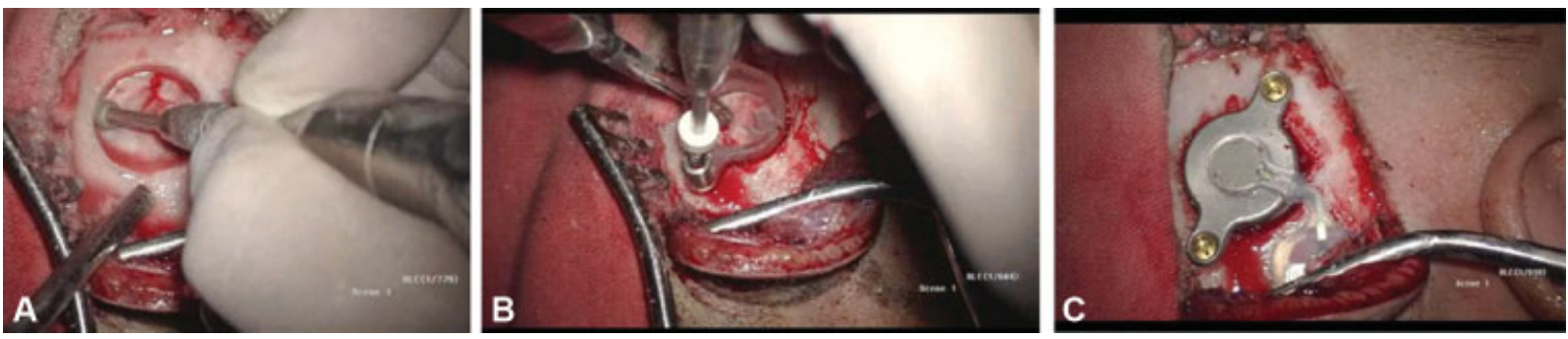

Fig. 2 (A) The dura is seen; the border should be smooth, (B): drilling of holes with a special burr, (C): implant in position and attached with screws.

the most horizontal axis position at the entrance of the EAC, where the eardrum is. The eardrum is the natural microphone of our ear, as mentioned before. Subsequently, the thickness of the flap is measured, always pointing to a thickness of $<7 \mathrm{~mm}$ for subsequent postsurgical coupling with the external processor. The bed is drilled for the BC-FMT until the dura is discovered (-Fig. 1C). At this point, it is necessary to drill carefully and to avoid compression maneuvers, due to the presence of the Labbé vein (subdural position and irregular route), which usually crosses the area where the BB will be placed.(-Fig. 2A) Posteriorly, two holes are made for the placement of the screws that will fix the implant and that transmit the vibrations generated by the electromagnetic coil of the BCI. ( - Fig. 2B) The edge of the surrounding bone of the implant bed must be smooth and separated from the dura to avoid tears or injuries of the dura.(-Fig. 2A) Finally, the BB is placed and the internal muscle flap is closed covering the prosthesis. Lastly, the skin flap is sealed with nonabsorbable suture ( - Fig. 2C). Head bandage is required. This technique does not offer major technical problems. Approximately after 30 days, the implant was activated by fitting.

\section{Audiological Assessments}

On the presurgical audiograms, the AC thresholds showed a PTA4 (0.5, 1,2 and $4 \mathrm{kHz}$ ) of $66.71 \mathrm{~dB} \mathrm{HL}$ (standard deviation $[\mathrm{SD}]= \pm 7.8$ ), while the bone conduction thresholds reached an average of $11.25 \mathrm{~dB}(\mathrm{SD}= \pm 6.9)$. The postoperative thresholds did not change at all. Four weeks after surgery, all the patients were fitted with the external processor. The audiological protocol was made. In the aided condition, the AC PTA 4 thresholds reached an average of $18.92 \mathrm{~dB}$ HL $(\mathrm{SD}= \pm 5.9)$.

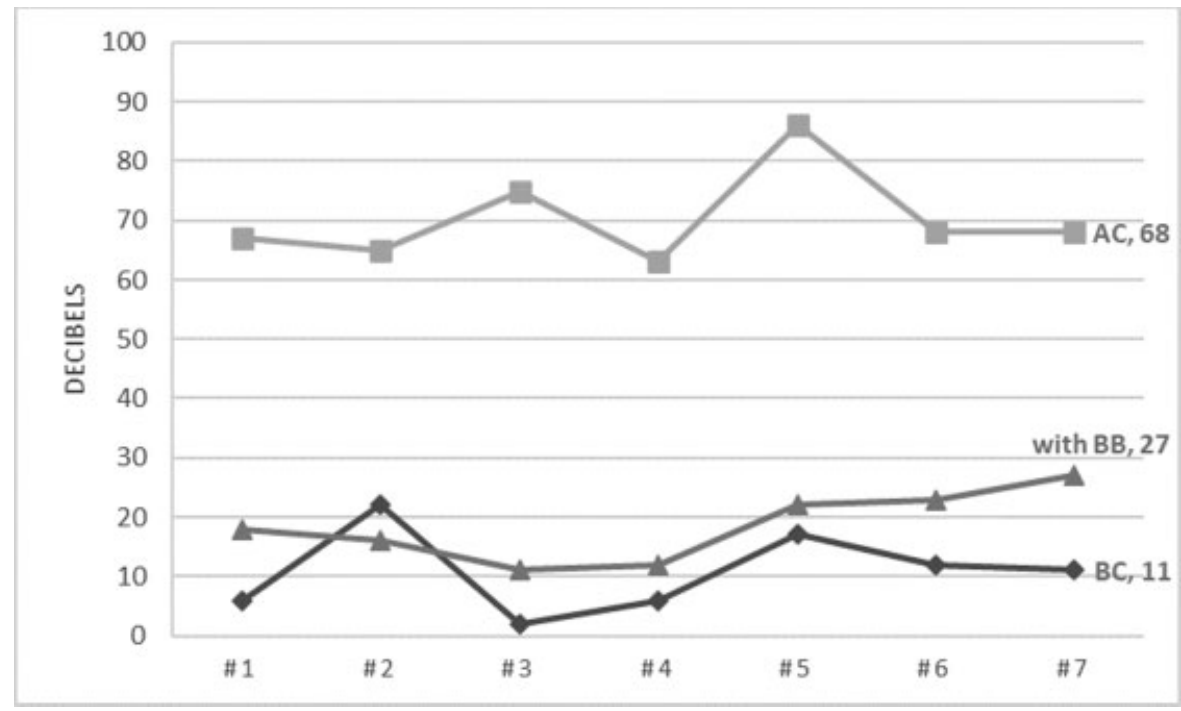

Fig. 3 PTA4 of patients with air and bone conduction thresholds, and aided with bone conductive implant. 


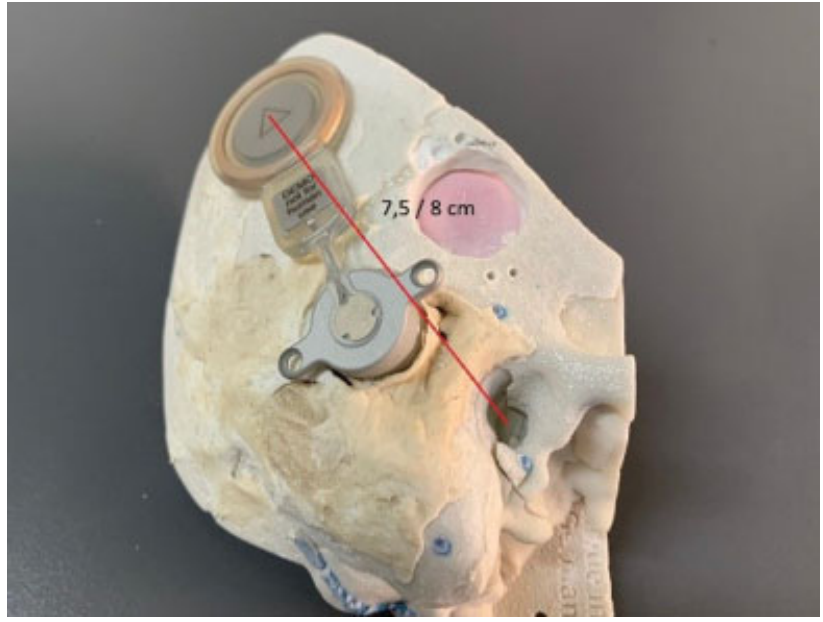

Fig. 4 Transmastoid approach: distance between magnet and the EAC.

The overall average percentage of speech discrimination before surgery was $37 \%(\mathrm{SD}= \pm 11.4)$. In the aided condition, it reached $91 \%$ in the $1^{\text {st }}$ month and $94 \%(S D= \pm 5.3)$ at 6 months, while WRS in noise (signal at $65 \mathrm{~dB}$ and noise at the same level: - 65dB - SON0) was $24 \%$ (SD = \pm 10.5 ) unaided and reached $74 \%(\mathrm{SD}= \pm 9.7)$ in aided condition. (-Fig. 3 )

\section{Complications}

Regarding complications, we did not have intraoperative complications. Postoperatively, only one patient mentioned some mild degree of skin irritation after 12 hours of use (it was the only patient without hair in between the magnet zone and the external processor).

\section{Discussion}

There are many papers that mention the auditory advantages of Bonebridge in conductive hearing loss, particularly in CAA. The present study examined the Bonebridge, the first active transcutaneous bone conduction implant system, implanted

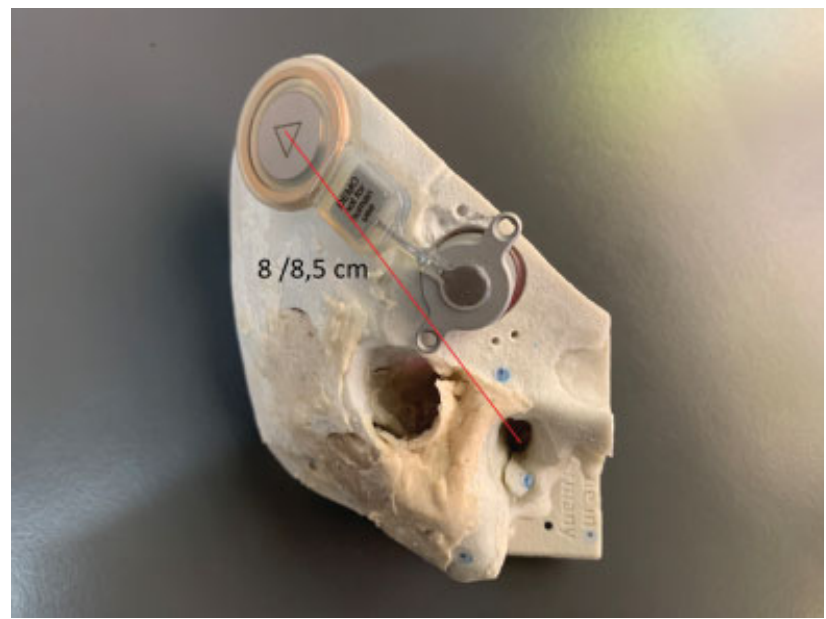

Fig. 5 Middle Fossa Approach, with usual position of the magnet showed the longest distance.

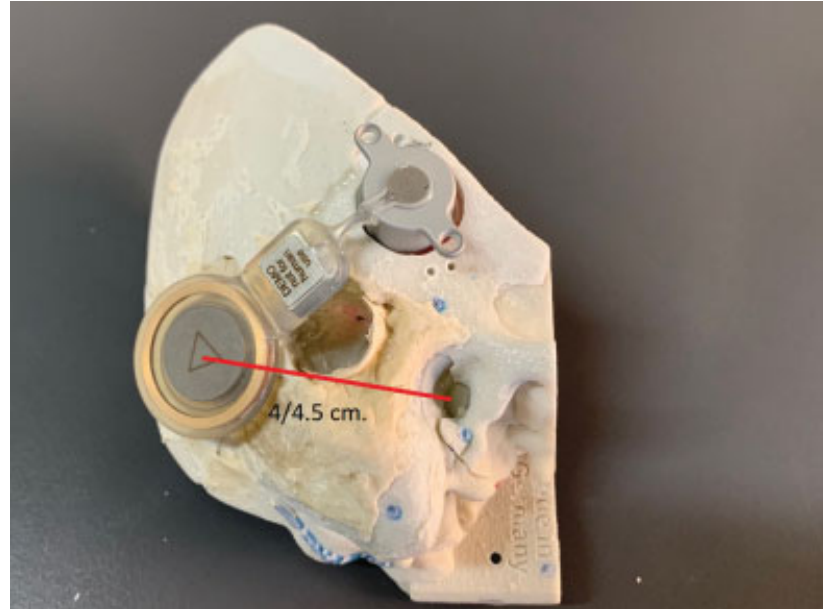

Fig. 6 Inverted Middle Fossa Approach showed the shortest distance from the EAC.

with a different approach. This is the first study of this novel approach where the results indicate audiological measurements comparable with other publications.

The idea was originated in a surgical laboratory doing different approaches and comparing the distance from the EAC to the position of the external audio processor (AP) as shown in -Figs. 4 and 5.

In the traditional approach (transmastoid), the distance between the EAC and the magnet is usually from 75 to $80 \mathrm{~mm}$, while in the middle fossa approach the distance is longer ( 80 to $85 \mathrm{~mm}$ ). In the inverted position, the distance reached 40 to $45 \mathrm{~mm}$ (-Fig. 6). Probably the half of distance would be benefit for localization and to reduce the shadow effect.

Therefore, the IMFA could be better for the audiological results, especially regarding localization and shadow effect.

Bravo-Torres et al implanted 15 children with congenital aural atresia in the middle fossa. Before surgery, the average AC threshold was $66.5 \mathrm{dBHL}$ (95\% confidence interval [CI]: 64.2-68.9), and with the BCHA switched on, the average threshold was $31.0 \mathrm{dBHL}$ (95\% CI: 28.2-33.8), decreasing to $25.2 \mathrm{dBHL}$ (95\%CI: 23.5-26.9) after a month of use. Therefore, the functional gain (FG) reached $41.3 \mathrm{dBHL}$. The average of correct speech recognition before surgery was $29.4 \%$ (95\% $\mathrm{CI}$ : 25.2-34.6), while in aided condition it increased to $90.7 \%$ (95\%CI: 87.4-93.9) and, a month afterwards, reached $96.4 \%$ (95\%CI: 92.7-100.2). ${ }^{7}$

Der et al (from the same group) implanted 24 patients with atresia with the same approach. The average AC thresholds before surgery were $66.5 \mathrm{dBHL}$ and $31 \mathrm{dBHL}$ when aided by bone conduction implant $(\mathrm{FG}=35.5 \mathrm{dBHL})$. The average speech recognition percentage before surgery was $29.4 \%$ (95\% CI 25.2-34.6), 90,7\% at the day of activation and reaching 96.4\% (95\% CI 92.7-100.2) aided after a 1-month follow-up. ${ }^{8}$

Baumgartner et al, in 12 pediatric patients, most of them (10 patients) affected by microtia/atresia, showed a preoperative mean monosyllabic word recognition score of $14.5 \%$ ( $\mathrm{SD}= \pm 21.6)$, which increased to $67.2 \% \quad(\mathrm{SD}= \pm 17.9)$ 1 month after implantation aided with Bonebridge, and to $82.1 \%(\mathrm{SD}= \pm 12.1)$ at 3 months. $^{9}$ 
In a previous paper, our group published a study in which fourteen children with CAC were implanted with Bonebridge. The preoperative PTA4 was $66.4 \mathrm{dBHL}$ (95\%CI: 64.2-68.6) and after activation moved to $19.2 \mathrm{dBHL}$ (95\%CI: 16.9-21.5), resulting in a mean functional gain of $47.2 \mathrm{dBHL}$. Regarding speech discrimination, the preoperative outcomes of the disyllabic measurements were $34.3 \%$, and for monosyllables they were $27.4 \%$. Following activation, the speech discrimination improved to $98.6 \%$ and $97.9 \%$, respectively. ${ }^{4}$

Magele et al recently published the first systematic review of use of BB. The meta-analysis for 30 conductive hearing loss subjects revealed a weighted functional gain of $39.48 \mathrm{~dB}$ SPL. Regarding the speech understanding in quiet, resulting in a mean unaided WRS score of $25.73 \pm 23.64 \%$ improving to $84.48 \pm 15.09 \%$ in the aided condition (overall mean improvement of almost $60 \%)^{10}$

However, the classic approaches sometimes are difficult, and very commonly produce compression of the sigmoid sinus in the mastoid approach or important bleeding in the retrosigmoid position. The IMFA overcomes these two problems. While it is sometimes necessary to compress the dura, it causes neither pain nor additional problems. In a paper by Lassaletta et al, this was extensively studied, and the pain was measured with Headache Impact Test (HIT6) and Pain Brief Inventory (BPI) prior and posterior to surgery and the level of pain did not change with or without compression of the dura. ${ }^{11}$

Finally, regarding complications, Sprinzl said that "Bonebridge offers a lower complication rate to percutaneous systems and higher and more reliable hearing gain compared to other transcutaneous or percutaneous systems." ${ }^{12}$ In our study, only one patient showed mild skin irritation.

\section{Conclusion}

The IMFA allows the nearest position of the microphone to the EAC. The technique is a suitable option to the 3 classical approaches with a similar rate of audiometric results and word speech discrimination. Due to the small number of subjects, more investigation is needed to determine the benefit of the novel approach in comparison with the others.

\section{Conflict of Interests}

The authors have no conflicts of interest to declare.

\section{References}

1 Gavilan J, Adunka O, Agrawal S, et al. Quality standards for bone conduction implants. Acta Otolaryngol 2015;135(12):1277-1285

2 Zernotti ME, Curet CA, Cortasa S, Chiaraviglio M, Di Gregorio MF. Congenital Aural Atresia prevalence in the Argentinian population. Acta Otorrinolaringol Esp 2019;70(01):32-35

3 Zernotti ME, Sarasty AB. Active Bone Conduction Prosthesis: Bonebridge(TM). Int Arch Otorhinolaryngol 2015;19(04): 343-348

4 Zernotti ME, Chiaraviglio MM, Mauricio SB, Tabernero PA, Zernotti M, Di Gregorio MF. Audiological outcomes in patients with congenital aural atresia implanted with transcutaneous active bone conduction hearing implant. Int J Pediatr Otorhinolaryngol 2019;119:54-58

5 Lassaletta L, Sanchez-Cuadrado I, Muñoz E, Gavilan J. Retrosigmoid implantation of an active bone conduction stimulator in a patient with chronic otitis media. Auris Nasus Larynx 2014;41 (01):84-87

6 Siegel L, You P, Zimmerman K, Parnes L, Agrawal SK. Active Transcutaneous Bone Conduction Implant: Audiometric Outcomes Following a Novel Middle Fossa Approach With SelfDrilling Screws. Otol Neurotol 2020;41(05):605-613

7 Bravo-Torres S, Der-Mussa C, Fuentes-López E. Active transcutaneous bone conduction implant: audiological results in paediatric patients with bilateral microtia associated with external auditory canal atresia. Int J Audiol 2018;57(01):53-60

8 Der C, Bravo-Torres S, Pons N. Active Transcutaneous Bone Conduction Implant: Middle Fossa Placement Technique in Children With Bilateral Microtia and External Auditory Canal Atresia. Otol Neurotol 2018;39(05):e342-e348

9 Baumgartner WD, Hamzavi JS, Böheim K, et al. A New Transcutaneous Bone Conduction Hearing Implant: Short-term Safety and Efficacy in Children. Otol Neurotol 2016;37(06):713-720

10 Magele A, Schoerg P, Stanek B, Gradl B, Sprinzl GM. Active transcutaneous bone conduction hearing implants: Systematic review and meta-analysis. PLoS One 2019;14(09):e0221484 Doi: 10.1371/journal.pone.0221484

11 Lassaletta L, Calvino M, Zernotti M, Gavilán J. Postoperative pain in patients undergoing a transcutaneous active bone conduction implant (Bonebridge). Eur Arch Otorhinolaryngol 2016;273(12): 4103-4110

12 Sprinzl GM, Wolf-Magele A. The Bonebridge Bone Conduction Hearing Implant: indication criteria, surgery and a systematic review of the literature. Clin Otolaryngol 2016;41(02):131-143 Doi: $10.1111 /$ coa. 12484 